\title{
Social media and the Canadian Journal of Public Health
}

\section{Les médias sociaux et la Revue canadienne de santé publique}

\author{
Daniel Fuller ${ }^{1} \cdot$ Louise Potvin ${ }^{2}$ \\ Published online: 28 February 2020 \\ (C) The Canadian Public Health Association 2020
}

Social media plays an integral role in the distribution and sharing of information (Public Media Alliance and United Nations, Education, Scientific and Cultural Organization 2017). Scientific journals cannot ignore the use of social media platforms in promoting their research ideas and staying connected with their communities worldwide. Many people are retrieving scientific information and news stories from social media. For the Canadian Journal of Public Health (CJPH) to flourish in today's society, we need to embrace social media as an intrinsic part of daily editorial, production, and communications processes (Public Media Alliance and United Nations, Education, Scientific and Cultural Organization 2017).

The CJPH Editorial Board is pleased to announce the new position of CJPH Social Media Associate Editor (SMAE), and to welcome Dr. Daniel Fuller to the position for a four-year term effective January 2020. Dr. Fuller and the CJPH Editors will be working with Emma Mallach, Communications Manager at the Canadian Public Health Association, and student volunteers from Memorial University of Newfoundland. We are excited about the opportunity to engage with researchers, decision-makers, public health practitioners, advocates, journalists, and others to share and discuss the latest in Canadian public health research, policy, and practice. We will focus our efforts on two social media platforms, Twitter and Facebook. Twitter is a social media platform familiar to many academics and an

Daniel Fuller

dfuller@mun.ca

1 School of Human Kinetics and Recreation, Memorial University of Newfoundland, St. John's, NL, Canada

2 École de Santé Publique - Département de Médecine Sociale et Préventive, Université de Montréal, Montréal, QC, Canada online home to many academic communities, including \#EpiTwitter and \#AcademicTwitter. Facebook is the largest social media platform and a good way to interact with those who may be less familiar with Twitter.

Our aim is to engage with you, public health researchers and practitioners, and to help promote your work. CJPH has had an active Twitter handle since October 2019. As of January 29, 2020, we had 683 followers. We will also create a Facebook page and begin to track metrics on Facebook. Our goals are to attract a significant number of followers on both platforms and to become a go-to place for current developments on public health innovations published in either of Canada's official languages (English or French).

We see our presence on social media as a way to engage further with the public health communities in Canada and the world, and to connect those communities that share CJPH as a go-to place for publishing their work and keeping informed. As a scientific journal, our communities are composed of authors, readers, and reviewers. Over the past few years, we have invested in ensuring that both researchers and practitioners feel welcome and well served in those communities. Social media is another means by which CJPH will take leadership in enabling these conversations and make public health research more relevant and accessible to practitioners, facilitating evidence-based public health practice. Likewise, we believe that social media can help identify pressing public health issues and trigger researcher interests.

We are excited to launch the Social Media Associate Editor position for the Canadian Journal of Public Health. If you have ideas, comments, or suggestions with regard to CJPH media or social media activities, please direct message us on Twitter or Facebook. You can follow us on Twitter @ CJPH_RCSP, and on Facebook (https://www.facebook.com/CJPH.RCSP/). Keep up with the hashtags, \#cjph_rcsp, \#cjph, and \#rcsp. You can 
follow Daniel Fuller on Twitter @ walkabilly. We look forward to engaging with you about the latest public health research, policy, and practice in Canada.

Daniel Fuller, PhD, Social Media Associate Editor, CJPH Louise Potvin, PhD, Editor-in-Chief

\section{Éditorial}

Les médias sociaux jouent un rôle essentiel dans la diffusion et le partage de l'information (Public Media Alliance and United Nations, Education, Scientific and Cultural Organization 2017). Les revues scientifiques ne peuvent pas négliger les plateformes de réseautage social lorsqu'il s'agit de promouvoir leurs idées de recherche et de rester en lien avec leurs communautés de par le monde. Beaucoup de gens vont chercher des informations et des reportages scientifiques dans les médias sociaux. Pour que la Revue canadienne de santé publique (RCSP) prospère dans la société d'aujourd'hui, nous devons profiter des médias sociaux et en faire un élément intrinsèque de nos processus de rédaction, de production et de communication quotidiens (Public Media Alliance and United Nations, Education, Scientific and Cultural Organization 2017).

Le comité éditorial de la RCSP a le plaisir d'annoncer la création du poste de " rédacteur associé, médias sociaux » (RAMS) et d'accueillir le professeur Daniel Fuller dans ce poste pour un mandat de quatre ans à compter de janvier 2020. Daniel Fuller et les rédacteurs de la RCSP travailleront avec Emma Mallach, gestionnaire des communications de l'Association canadienne de santé publique, et avec des bénévoles étudiants de l'Université Memorial de TerreNeuve. Nous sommes heureux de la possibilité de créer un contact avec des chercheurs, des décideurs, des praticiens de la santé publique, des militants, des journalistes et d'autres pour parler des actualités de la recherche, des politiques et de la pratique en santé publique au Canada. Nous concentrerons nos efforts sur deux plateformes de réseautage social : Twitter et Facebook. Twitter est une plateforme connue dans les milieux universitaires et un toit en ligne pour de nombreuses communautés du monde de l'enseignement, dont \#EpiTwitter et \#AcademicTwitter. Facebook, la plus grande plateforme de réseautage social, représente un bon moyen d'interagir avec les personnes qui connaissent moins Twitter.

Notre objectif est de dialoguer avec vous, chercheurs et praticiens de la santé publique, et de vous aider à faire connaitre votre travail. La RCSP a un identifiant Twitter actif depuis octobre 2019. Au 29 janvier 2020, nous avions 683 abonnés. Nous créerons aussi une page Facebook et commencerons à suivre des indicateurs sur Facebook. Nous voulons attirer un nombre important d'abonnés sur ces deux plateformes et devenir un site incontournable pour qui veut connaître les dernières innovations en santé publique publiées dans l'une ou l'autre des langues officielles du Canada (français ou anglais).

Nous voyons notre présence dans les médias sociaux comme un moyen de mieux communiquer avec les communautés de la santé publique du Canada et du reste du monde, et de relier entre elles les communautés qui se servent de la RCSP pour publier leurs travaux et se tenir au courant. Comme nous sommes une revue scientifique, nos communautés se composent d'auteurs, de lecteurs et d'évaluateurs. Ces dernières années, nous avons investi pour que tant les chercheurs que les praticiens se sentent bien accueillis et bien servis dans ces communautés. Les médias sociaux sont un autre moyen pour la RCSP de prendre l'initiative de permettre de telles conversations et de rendre la recherche en santé publique plus pertinente et plus accessible pour les praticiens, ce qui facilitera la pratique d'une santé publique fondée sur les preuves. Réciproquement, nous croyons que les médias sociaux peuvent contribuer à repérer les problèmes de santé publique pressants et à susciter l'intérêt des chercheurs.

Nous sommes ravis de créer le poste de rédacteur associé, médias sociaux de la Revue canadienne de santé publique. Si vous avez des idées, des commentaires ou des suggestions concernant les activités de la RCSP dans la presse traditionnelle ou les médias sociaux, veuillez nous envoyer un message sur Twitter ou Facebook. Vous pouvez nous suivre sur Twitter @CJPH_RCSP et sur Facebook à https:// www.facebook.com/CJPH.RCSP/. Consultez les mots-clics \#cjph_rcsp, \#cjph et \#rcsp. Vous pouvez aussi suivre Daniel Fuller sur Twitter@walkabilly. Nous avons hâte de nous entretenir avec vous des dernières nouvelles dans le monde de la recherche, des politiques et de la pratique en santé publique au Canada.

Daniel Fuller, Ph. D., rédacteur associé, médias sociaux, RCSP Louise Potvin, Ph. D., rédactrice en chef

\section{Reference/Référence bibliographique}

Public Media Alliance and United Nations, Education, Scientific and Cultural Organization. (2017). Social Media Guidelines. http:// www.unesco.org/new/fileadmin/MULTIMEDIA/FIELD/Kingston/ Social-Media-Guideline-A-Handbook-2017_01.pdf. Accessed January 9, 2020.

Publisher's note Springer Nature remains neutral with regard to jurisdictional claims in published maps and institutional affiliations. 